\title{
Ritmo de crecimiento y longevidad de follaje del estrato arbustivo en la Reserva Biológica A. Brenes, Costa Rica
}

\author{
Elke Freiberg y Martin Freiberg \\ Departamento Spezielle Botanik, Universidad de Ulm, 89069 Ulm, República Federal de Alemania, Fax: ++49-731- \\ 502-2720, correo electrónico: elke.freiberg@ biologie.uni-ulm.de
}

\begin{abstract}
During one and a half years (February 1991 - July 1992) the growth rhythm and the longevity of leaves of 22 abundant understory species in the tropical premontane rain forest of Reserva Biológica Alberto Ml. Brenes, Alajuela, Costa Rica, were investigated. Three types of growth rhythm were identified. Most species, especially the monocotyledones, produce new leaves in defined intervals throughout the year. Only some have a slower leaf production in the dry season. Other species sprout periodically or only once a year. This interval, which is the time period between the beginning of the shooting of one leaf and the next, varied among the investigated species between 17-22 days (Malvaviscus arboreus, Urera elata, Myriocarpa longipes) and more than half a year (Cyclanthus bipartitus, Iriartea deltoidea, Ocotea gomezii). For some species this interval was longer at dry and dark sites (M. arboreus), but in many cases microclimatical factors such as temperature, relative light intensity, and evaporation varied to such a small extent within the forest that the differences did not lead to significant influences on growth factors of the plants. The mean leaf longevity varied between a half and four years. The maximum leaf age of the palms Cryosophila warscewiczii, I. deltoidea and Calyptrogyne trichostachys was estimated to be more than five years. In some cases leaf age was significantly correlated with microclimatical factors. Leaves of Begonia cooperi growing at higher light intensities in gaps fell earlier than leaves of plants in darker sites in the understory. Generally, the longevity of leaves was lower in plants growing at moister sites, but in most cases this relation was not significant. The data show that even in a rather constant climate in the understory of a very moist premontane rain forest, there is some seasonality in growth rhythm and that plant growth varies between different sites within the forest although climate variations are very small.
\end{abstract}

Key words: Costa Rica, growth rhythm, longevity of leaves, microclimate, phenology, seasonality, tropical premontane rain forest, understory.

En los bosques templados el frío en invierno causa la abscisión de las hojas, mientras que en los bosques tropicales el ritmo de precipitación tiene más importancia. El aspecto de bosques tropicales muy húmedos es perennifolia. Pero, aunque el clima es casi constante durante todo el año, ciertas especies muestran un ritmo anual de fenología (Medway, citado en Whitmore 1993, p. 73). Por ejemplo, en la estación científica "La Selva", Costa Rica, mu- chos árboles brotan hojas nuevas al principio de la estación seca, poco después de la abscisión de todas las hojas viejas (Frankie et al. 1974). Allá, se distinguen tres tipos de brotación (Frankie et al. 1974):

I: especies que producen hojas nuevas contínuamente y que no muestran una época distinta de abscisión (26\% de las especies del dosel, $37 \%$ de las especies del estrato arbustivo), 
II: especies que cambian entre la producción de muchas, pocas o ninguna hojas y que no muestran una época distinta de abscisión o una época sin hojas $(37 \%, 44 \%)$,

III: y especies con épocas distintas de brotación y abscisión $(25 \%, 7 \%)$.

En bosques tropicales con una estación seca más distintiva que en "La Selva", como en la Isla Barro Colorado, Panamá, una gran cantidad de árboles son caducifolios y botan todas las hojas al principio de la estación seca y no tienen hojas antes de las primeras lluvias (Leigh y Windsor 1982). Otros árboles del mismo bosque son perennifolios o cambian las hojas muy irregularmente e imprevisiblemente, como Ceiba pentandra. Un cambio periódico de hojas se observa especialmente en el dosel, mientras que las plantas del estrato arbustivo son perennifolios (Richards 1966).

En bosques tropicales secos, como en Guanacaste, Costa Rica, la mayoría de los árboles son caducifolios y pierden sus hojas cada año durante la estación seca para reducir la evaporación (Frankie et al. 1974, Reich y Borchert 1984). Plantás que crecen en sitios más húmedos cerca de ríos en el mismo bosque se permanecen verdes o el período defoliado es más corto (Frankie et al. 1974, Janzen 1975, Reich y Borchert 1984). Además, ciertas especies cambian su hábito a diferentes altitudes en Costa Rica (Borchert 1983). En sitios más altos la intensidad de la sequía disminuye y especies como Erythrina poeppingiana y Tabebuia rosea cambian de un aspecto caducifolio a un aspecto perennifolio. Mientras que $T$. rosea se queda sin hojas durante varias semanas en Guanacaste, en alturas más altas árboles de la misma especie tienen hojas nuevas al mismo tiempo que botan las hojas viejas (Borchert 1983).

Otros factores climáticos como temperatura, viento y factores edáficos pueden influir en la producción y caída de las hojas (Addicott y Lyon 1973, citado en Bentley 1979).

La longevidad de las hojas varía mucho (Chabot y Hicks 1982). Las hojas de efímeros viven solamente pocas semanas, mientras que las hojas de una Araucaria pueden vivir por 25 años. Las hojas de Welwitschia mirabilis crecen durante toda la vida de la planta. Mientras que una gran cantidad de los árboles en los bosques tropicales cambian sus hojas por lo menos una vez cada año, la edad promedio de las hojas en el estrato arbustivo en el Parque Nacional Braulio Carillo, Costa Rica, varía entre 14-15 meses y 5-6 años (R. Lücking 1994, A. Lücking 1995). Un factor que puede influir en la longevidad de follaje es la luz (Bentley 1979). En la Isla Barro Colorado, Panamá, la edad promedio de hojas de especies típicas de sombra es tres veces más alta (21.7 meses) que la edad de las hojas de especies de claros (6.8 meses) (Coley 1988).

Además, el método de defenderse de los herbívoros puede influir en la longevidad de las hojas (Coley 1988). Plantas con hojas longevas producen sustancias de defensa de peso molecular grande, como taninos y ligninas. Las hojas producen estas sustancias solamente una vez, y así tienen gastos solamente al principio de su vida. Plantas con hojas de vida corta producen sustancias de menor peso molecular, como alcaloides y monoterpenos, los cuales se sintetizan contínuamente (Coley 1988).

El objetivo de este estudio es analizar el ritmo de defoliación y brotación y determinar la longevidad de follaje de diferentes plantas en el estrato arbustivo en la Reserva Biológica Alberto M1. Brenes, San Ramón, Costa Rica. Además, se quería verificar la existencia y el efecto del clima sobre la fenología de las plantas en un bosque húmedo premontano. El conocimiento de la fenología tiene importancia para investigaciones sobre los organismos en la filósfera (E. Freiberg 1998) o sobre los ciclos de minerales.

\section{MATERIALES Y MÉTODOS}

Área de investigación: Las investigaciones se realizaron alrededor de la estación biológica de la Reserva Biológica Alberto Ml. Brenes (RBAMB) en la Provincia Alajuela, San Ramón, Costa Rica. La reserva está situada en la Cordillera de Tilarán en una altura de 
800 a 1500 m s.n.m. $\left(10^{\circ} 10^{\prime}-10^{\circ} 24^{\prime}\right.$ N; $84^{\circ} 34^{\prime}-84^{\circ} 40^{\prime}$ W). En su mayoría, el bosque es primario. Se encuentran transiciones de bosque tropical muy húmedo, bosque premontano muy húmedo a bosque montano bajo muy húmedo (Holdridge 1967, Hartshorn 1983, Vargas 1991). Ya existen varias investigaciones sobre la vegetación (Ortiz 1991, Wattenberg y Breckle 1995, 1997, M. Freiberg 1996). Se midió una precipitación anual de $4360 \mathrm{~mm}$ desde mayo 1991 hasta abril 1992 (E. Freiberg 1994, M. Freiberg 1996) y $3660 \mathrm{~mm}$ desde setiembre 1992 hasta setiembre 1993 (Dombeck 1997). El clima era perhúmedo (según terminología de Walter 1973) desde abril hasta diciembre y había una estación seca desde enero hasta marzo. Al mismo tiempo, la temperatura media mensual en el estrato arbustivo a $1.5 \mathrm{~m}$ sobre el suelo varió entre $18.0^{\circ} \mathrm{C}$ y $20.8^{\circ} \mathrm{C}$ (M. Freiberg 1997).

Plantas investigadas: Se investigaron 22 especies de plantas vasculares, las cuales fueron abundantes en el estrato arbustivo cerca de la estación biológica a una altura de 870 m s.n.m. (Cuadro 1). Se depositó material herborizado de cada especie en el Herbario Nacional de Costa Rica (CR) y en el Herbario de la Universidad de Ulm, Alemania (ULM), bajo los números que se muestran en Cuadro 1.

\section{CUADRO 1}

Lista de las plantas investigadas, número de colección depositada en los herbarios (CR/ULM) y tipo de brotación: anual $(A)$, contínua $(C)$ y periódica $(P)$.

List of investigated plants, number of collection deposited in the herbaria (CR/ULM) and type of leaf production: annually $(A)$, continuously $(C)$, and periodically $(P)$.

Posición taxonómica

Especie

\section{Pteridophyta \\ Hypolepidiaceae \\ Monocoty edoneae \\ Arecaceae}

\section{Cyclanthaceae \\ Araceae}

He iconiaceae

\section{Marantaceae}

Dicotyledoneae

Acanthaceae

Begoniaceae

Lauraceae

Malvaceae

Melastomataceae

Monimiaceae

Piperaceae

Rubiaceae

Urticaceae
Pteris sp.

Calyptrogyne trichostachys Burret

Chanaedorea tepejilote Liebm.

$=$ C. exorrhiza H.A.Wendl. ex Guillaumin

Cryosophila warscewiczii (H.A.Wendl.)

Burret $=$ C. albida Bartlett

Iriartea deltoidea Ruiz \& Pav.

$=\mathrm{I}$. gigantea $\mathrm{H}$. A. Wendl.

Cyclanthus bipartitus Poit.

Philodendron verrucosum Mathieu

Heliconia irrasa ssp. undulata Daniels

$\&$ Stiles

Calathea leucostachys Hook. f.

Justicia crenata (Leonard) Durkee

Spathacanthus hoffmannii Lindau

Begonia cooperi C.DC.

Ocotea gomezii W.C.Burger

Malvaviscus arboreus Cav.

Blakea gracilis Hemsl.

Siparuna nicaraguensis Hemsl.

Piper augustum Rudge

Piper sp.

Hoffmannia dotae Standl.

Psychotria cf. uliginosa Sw.

Myriocarpa longipes Liebm.

Urera elata (Sw.) Griseb.
Número en

el herbario

CR / ULM

$$
\text { 234/- }
$$

207/92207

.192304

$-/ 92218$

$-192224$

$-192212$

$306 /-$

Tipo de brotación

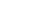

$109 / 91109$

$317 / 91317$

$104 / 92104$

$-/ 92223$

$102 / 91335$

$336 / 91336$

106

$-/ 91213$

$111 / 92111$

100/91330

101/-

$303 / 92303$

$333 / 91334$
C

C

C

C

C

C

C

C

C
C
C
A
P
P
P
P
C
C
C
C
P 
Determinación del ritmo de crecimiento y de la edad de follaje: Durante un estudio de un año y medio (febrero 1991-julio 1992) se marcaron las hojas recién brotadas de las 22 especies con anillos plásticos. Al final de los estudios se calculó la edad de cada hoja marcada y el intervalo promedio entre el desarrollo de dos hojas siguientes, lo que fue denominado "frecuencia de brotación", para cada planta o eje (Fig. 1). Además, se determinó la cantidad de nudos entre el punto y la hoja más antigua de cada eje. Sobre la base de estos datos se estimó la edad de las hojas que no fueron marcadas y la longevidad de follaje promedio y máxima de cada especie, asumiendo condiciones constantes.

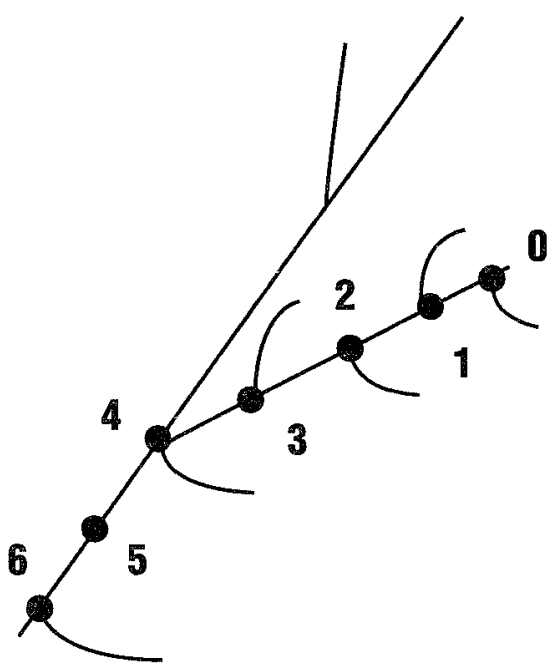

Fig. 1. Determinación de la longevidad de las hojas y la frecuencia de brotación. $0=$ hoja que empieza a brotar; 1 = hoja de 30 días; 2 = hoja de 60 días; 3 = hoja de 90 días; $4=$ hoja de 120 días; 5 = hoja ya se ha caido; $6=$ la hoja más vieja de la eje. El intervalo promedio entre dos hojas siguientes es 30 días, la cantidad de los nudos es 6 , y la longevidad de la hoja 6 es 180 días (30 días por 6 nudos).

Mediciones climáticas: Al mismo tiempo, se midieron factores microclimáticos (la temperatura, la intensidad de la luz, la evaporación, y la precipitación) para determinar si el microclima influye en el ritmo de crecimiento.

Se midió la intensidad de la luz con un fotómetro (LX-101, Lutron, Taiwan) directamente encima de cada hoja y simultáneamente enci- ma del dosel y se calculó la iluminación relativa. Se realizaron las mediciones de la temperatura con termómetros de alcohol contínuamente en 16 sitios en el área de investigación. En estos sitios también se midió la evaporación con evaporímetros Piché con papel de filtro de $3 \mathrm{~cm}$ de diámetro (Bannister 1986). Sobre la base de estos datos se subdividió el área de investigación en tres clases de humedad: 9-14.9 $\mu \mathrm{l} \mathrm{H2O} \mathrm{h-1}$ (clase 1), 15-20.9 $\mu \mathrm{l} \mathrm{H2O} \cdot \mathrm{h}-1$ (clase 2), y 21$26.9 \mu \mathrm{l} \mathrm{H} 2 \mathrm{O}$ h-1 (clase 3). Se midió la precipitación cada día cerca de la estación biológica.

\section{RESULTADOS}

El ritmo de crecimiento: Se distingió entre tres tipos de brotación: la brotación contínua, la brotación periódica y la brotación anual (Cuadro 1). Las hojas de algunas especies, especialmente de las monocotiledóneas, brotaron contínuamente durante todo el año. Otras especies, como Malvaviscus arboreus y Urera ela$t a$, produjeron series de hojas muy rápidamente y después no lo hicieron durante cierto tiempo. Ocotea gomezii produjo hojas nuevas solamente una vez cada año, en diciembre. Todas las plantas investigadas fueron de aspecto perennifolio y nunca se permanecieron sin hojas.

Entre las especies, también varió la frecuencia de brotación, es decir el intervalo promedio entre el desarollo de dos hojas siguientes. Algunas plantas produjeron las hojas rápidamente. Por ejemplo, una rama de Myriocarpa longipes tuvo una hoja nueva cada 25 días (Cuadro 2), mientras que otras especies, como Iriartea deltoidea y Cyclanthus bipartitus, solamente produjeron una o dos hojas cada año.

La cantidad de nudos con hojas de los ejes o las plantas varió entre tres (Chamaedorea tepejilote) y más de 30 (M. arboreus y M. longipes) (Cuadro 2).

Longevidad de follaje: La longevidad de las hojas varió distintamente (Cuadro 2). Entre las plantas investigadas, Calyptrogyne trichostachys, Cryosophila warscewiczii, C. bipartitus, I. deltoidea y $O$. gomezii tuvieron las hojas más viejas. Según la extrapolación de los datos, las 


\section{CUADRO 2}

La frecuencia de brotación de 22 especies del estrato arbustivo como intervalo promedio entre dos hojas, el número de nudos desde la hoja más vieja y el punto del eje, la longevidad de follaje promedio y máxima y el número de ejes o plantas analizadas, ordenado por la columna 2

The frequency of leaf production of 22 understory species as mean interval between two leaves, the number of nodes between the oldest leaf and the shoot tip, the mean and maximum longevity of leaves, and the number of shoots or plants that were considered in the analysis, ordered by the second column

$\begin{array}{cc}\begin{array}{c}\text { Intervalo } \\ \text { promedio } \\ \text { [días] }\end{array} & \begin{array}{c}\text { Cantidad } \\ \text { de nudos }\end{array} \\ \end{array}$

Especie

Promedio Máxima

\section{Longevidad de follaje} [meses]

Promedio Máxima

M. arboreus

$U$. elata

M. longipes

B. cooperi

S. nicaraguensis

$P$. augustum

C. leucostachys

H. dotae

Piper sp.

B. gracilis

$P$. cf. uliginosa

J. crenata

$P$. verrucosum

C. tepejilote

$S$. hoffmannii

H. irrasa ssp. undulata

C. trichostachys

C. warscewiczii

Pteris sp.

C. bipartitus

I. deltoidea

o. gomezii
17

\section{2}

25

31
34

45

47

52

58

59

66

68

71

107

126

108

132

147

164

172

266

360

$\begin{array}{rr}18 & 35 \\ 14 & 27 \\ 20 & 39 \\ 8 & 12 \\ 6 & 8 \\ 8 & 11 \\ 6 & 6 \\ 9 & 16 \\ 8 & 10 \\ 4 & 7 \\ 9 & 10 \\ 4 & 9 \\ 5 & 7 \\ 3 & 5 \\ 5 & 12 \\ 6 & 6 \\ 11 & 14 \\ 10 & 14 \\ 3 & 5 \\ 8 & 10 \\ 6 & 7 \\ 5 & 5\end{array}$

hojas de I. deltoidea podrían tener más de cinco años. Normalmente, las hojas de Begonia cooperi, Siparuna nicaraguensis, Blakea gracilis, Justicia crenata, U. elata, $M$. arboreus y Calathea leucostachys no tuvieron más de un año.

Hubo una relación significativa entre la longevidad de la hoja y la frecuencia de la brotación $(n=22$, coeficiente de corelación de Spearman, $\mathrm{P}<0.001$, Fig. 2). Sin embargo hubo excepciones. Por ejemplo, las hojas de C. trichostachys, C. warscewiczii y M. longipes brotaron relativamente rápido pero alcanzaron bastante edad, mientras que las hojas de C. tepejilote y Pteris sp. tuvieron relativamente una corta vida pero brotaron muy despacio. No hubo una relación significativa entre la cantidad de nudos y la longevidad de follaje $(\mathrm{n}=22$, Spearman, $\mathrm{P}=0.946)$ ni con la frecuencia de brotación $(n=22$, Spearman, $P=0.982)$.

$\begin{array}{rrrr}35 & 10 & 20 & 16 \\ 27 & 10 & 20 & 17 \\ 39 & 17 & 32 & 19 \\ 12 & 8 & 12 & 14 \\ 8 & 7 & 9 & 7 \\ 11 & 12 & 17 & 9 \\ 6 & 9 & 9 & 1 \\ 16 & 16 & 28 & 24 \\ 10 & 15 & 19 & 15 \\ 7 & 9 & 14 & 13 \\ 10 & 19 & 22 & 2 \\ 9 & 10 & 20 & 23 \\ 7 & 12 & 17 & 3 \\ 5 & 12 & 18 & 11 \\ 12 & 21 & 50 & 41 \\ 6 & 22 & 22 & 1 \\ 14 & 50 & 62 & 3 \\ 14 & 49 & 69 & 5 \\ 5 & 18 & 27 & 8 \\ 10 & 46 & 57 & 7 \\ 7 & 53 & 62 & 6 \\ 5 & 54 & 54 & 1\end{array}$

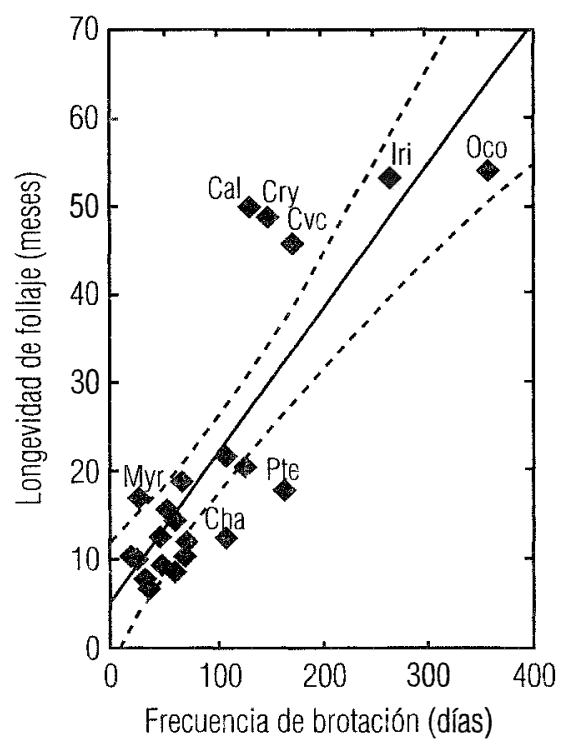

Fig. 2. Relación entre la frecuencia de brotación de las hojas y la longevidad de las hojas. 
Influencia del microclima: Aunque el clima pareció ser muy constante en el estrato arbustivo del área de investigación, se pudo distinguir sitios más o menos húmedos y más o menos oscuros. Generalmente, la frecuencia de brotación $(M$. arboreus $(\mathrm{n}=310$, Spearman, $\mathrm{P}=0.0002$, Fig. 3) y Hoffmannia dotae $(\mathrm{n}=147$, Spearman, $\mathrm{P}=0.0047)$ ) y la longevidad de follaje $(M$. arboreus $(\mathrm{n}=13, \quad$ Spearman, $\mathrm{P}=0.0388)$ y $B$. cooperi $(\mathrm{n}=14$, ANOVA de Kruskal-Wallis, $\mathrm{P}=0.0014$, Fig. 4)) fueron más largos en sitios más oscuros, pero esta relación fue significante solamente en pocos casos.

La longevidad de follaje generalmente fue más larga en sitios más secos. Esta relación fue significante para $M$. longipes $(n=13$, MannWhitney U-Test, $\mathrm{P}=0.0425$, Fig. 4). La frecuencia de brotación de $B$. cooperi $(\mathrm{n}=117$, U-Test, $\mathrm{P}=0.0002), \quad H$. dotae $(\mathrm{n}=147$, U-Test, $\mathrm{P}=0.0119), M$. arboreus $(\mathrm{n}=310$, ANOVA de Kruskal-Wallis, $\mathrm{P}=0.0007$, Fig. 3) y $B$. gracilis $(\mathrm{n}=45$, U-Test, $\mathrm{P}=0.0039)$ fue significantemente más corto en sitios más húmedos. Con solamente una excepción, la cantidad de nudos se mostró independiente de la humedad y de la luz.

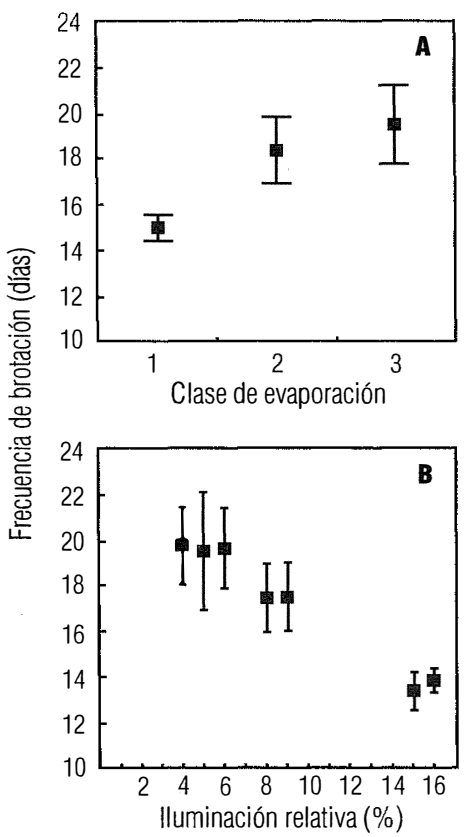

Fig. 3. Influencia de la humedad (clase de evaporación 1 es más húmedo que clase 3) (A) y de la iluminación relativa (B) sobre el crecimineto de las hojas de $M$. arboreus.

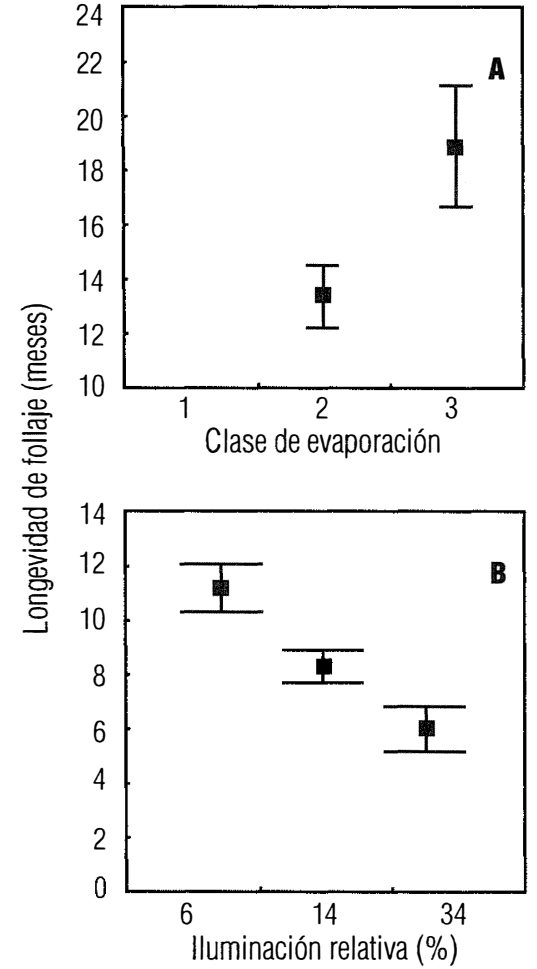

Fig. 4. Influencia de la humedad (clase de evaporación 1 es más húmedo que clase 3) (A) y de la iluminación relativa (B) sobre la longevidad de las hojas de $M$. longipes (A) y B. cooperi (B).

La diferencia promedio entre la temperatura más alta y más baja medida simultáneamente en los 16 sitios fue solamente $1.2^{\circ} \mathrm{C}$. Por eso, se supuso que en esa región la temperatura no tuvo por consecuencia diferencias en el ritmo de crecimiento de las hojas.

Influencia de la precipitación: Entre las plantas de tipo de brotación contínuo, el ritmo anual de la precipitación influyó la frecuencia de brotación de algunas especies (Fig. 5). En la estación seca el intervalo entre dos hojas de $M$. longipes, B. cooperi, C. trichostachys y de Spathacanthus hoffmannii fue más largo que en la estación lluviosa. Lo mismo vale para $H$. dotae $(\mathrm{n}=14$, Spearman, $\mathrm{P}=0.002)$. Por otro lado, la frecuencia de brotación de $C$. warscewiczii y $C$. tepejilote fue constante durante todo el año. 
Chamaedorea tepejilote

$n=14 ; P=0.070$

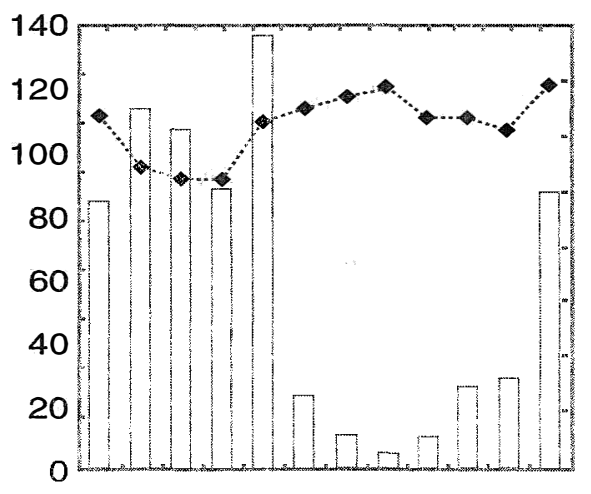

Calyptrogyne trichostachys

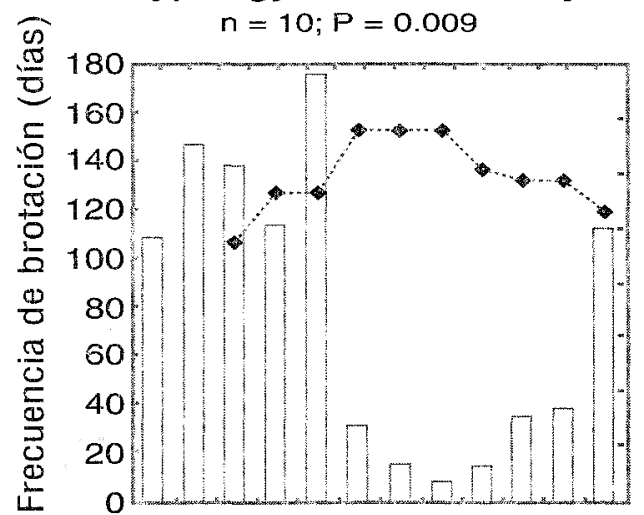

Myriocarpa longipes

$\mathrm{n}=14 ; \mathrm{P}=0.004$

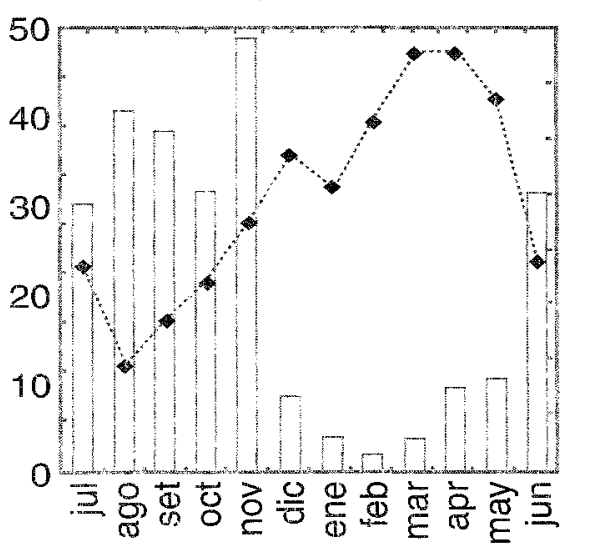

Crysosphila warscewiczii $n=10 ; P=0.243$

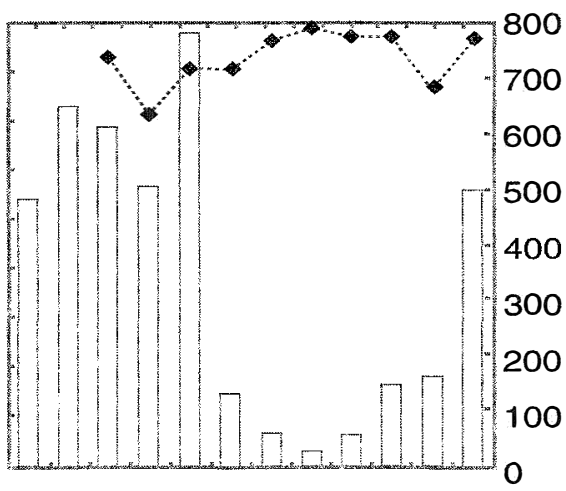

Spathacanthus hoffmannii $\mathrm{n}=14 ; \mathrm{P}=0.005$

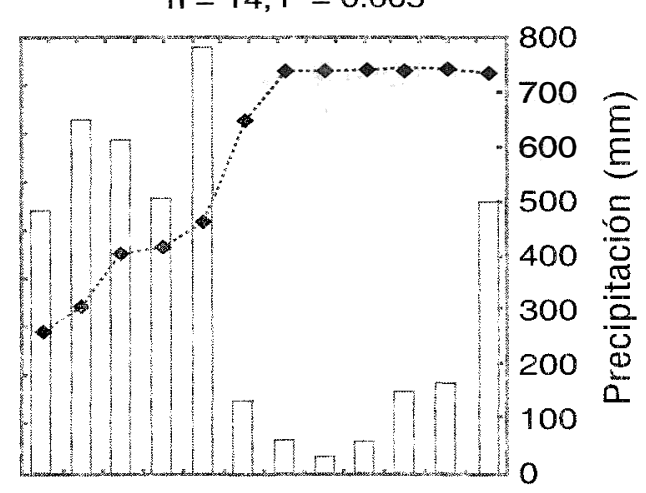

Begonia cooperi

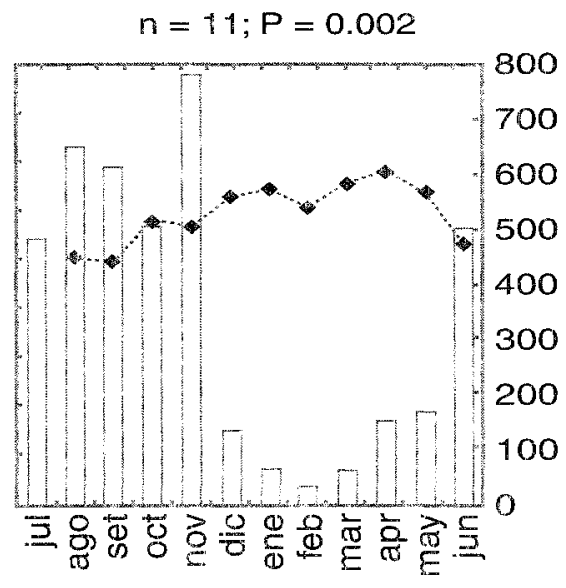

Meses en 1991 y 1992

Fig. 5. Relación entre la frecuencia de brotación de las hojas (lineas) y la precipiación mensual (columtas) desde julio 1991 hasta junio 1992. $\mathrm{n}=$ número de meses analizados, $\mathrm{P}<0.05$ es signilicante. 


\section{DISCUSIÓN}

La mayoría de las plantas investigadas en el sotobosque de la RBAMB brotaron las hojas contínuamente durante todo el período del estudio: tipo de brotación C o tipo I según Frankie et al. (1974), aunque algunas de estas especies (C. trichostachys, S. hoffmannii, M. longipes, $B$. cooperi, $H$. dotae) crecen más despacio en la estación seca. Muchas palmas producen hojas contínuamente, como Coco nucifera, que produce una hoja nueva y bota una hoja vieja de cinco años cada mes (Mabberly 1983). También Zamia skinneri produce hojas nuevas en cualquier mes, pero con más frecuencia al principio de la estación lluviosa (Clark et al. 1992). Otras especies brotan las hojas en períodos irregulares: tipo $\mathrm{P}$ o tipo II según Frankie et al. (1974). Ninguna de las especies del estrato arbustivo pertenece al tipo III según Frankie et al. (1974), sin embargo un árbol del dosel, Ficus jimenezii, pierde todas las hojas al principio de la estación seca en diciembre y enero y tiene hojas nuevas poco después en enero y febrero (M. Freiberg 1994).

También, la frecuencia de brotación varía entre las plantas investigadas. Algunas especies producen de 15 a 21 hojas nuevas (M. longipes, $U$. elata, $M$. arboreus), otras solamente de una a dos hojas cada año (I. deltoidea, $O$. gomezii). Estudios en la estación biológica "La Selva", Costa Rica (Bentley 1979, Clark et al. 1992), muestran que las especies en el estrato arbustivo producen de cinco a seis hojas por eje cada año (Syngonium sp., Parathesis chryophylla, Renealmia cernua) y otras solamente de una a dos (Myrica carnea, Inga thibaudiana, Aphelandra sp., Proteum pittieri, Zamia skinneri).

La longevidad de las hojas varía distintamente entre las plantas en el bosque tropical premontano de la RBAMB. Algunas plantas no tienen hojas de más de nueve o diez meses y otras hojas, según la extrapolación, pueden vivir más de cinco años. Este resultado coincide con estudios sobre la fenología de las hojas en el estrato arbustivo que se realizaron en el Parque Nacional Braulio Carillo, Costa Rica, (R.
Lücking 1994, A. Lücking 1995). Allá, la longevidad promedio de las hojas varía entre 14 a 15 meses (Costus curvibracteatus, Costus malortieanus, Columnea consanguinea) y de 63 a 73 meses (Ocotea atirrensis, Naucleopsis naga, I. deltoidea). En ambas regiones, entre las plantas investigadas, I. deltoidea y especies de Ocotea son las plantas con las hojas más viejas. Valores similares también existen sobre las epífitas vasculares en RBAMB. Columnea microphylla tiene hojas de más de cuatro años, mientras que Columnea verecunda no tiene hojas de más de un año (M. Freiberg 1994). En la estación biológica "La Selva", Costa Rica, las hojas de Zamia skinneri pueden vivir nueve años o más (Clark et al. 1992).

La longevidad depende en parte del microclima. Generalmente, la longevidad de follaje fue más alta en sitios más oscuros y más secos. Pero, esta relación no fue significante en todos los casos. Las variaciones del microclima (temperatura, luz, humedad) en el sotobosque son relativamente pequeñas y muchas veces no tienen por consecuencia una influencia significante sobre el crecimiento. Si los estudios incluyen individuos que crecen en claros, como en el caso de B. cooperi, existe una correlación significante. Este resultado coincide con estudios realizados en la Isla Barro Colorado, Panamá (Coley 1988). Allá, la longevidad promedio de hojas de especies típicas de sombra es tres veces más alta, 21.7 meses, que la longevidad de las hojas de especies de claros, 6.8 meses (Coley 1988). En "La Selva", Bentley (1979) también encontró, que las plantas en los claros botan sus hojas más rápido que en la sombra. Otros estudios también muestran que la abscisión de las hojas de plantas creciendo en la luz es más rápida que la abscisión de hojas en la sombra (Bentley 1979, Ackerly y Bazzaz 1995).

Esta diferencia entre las plantas creciendo en la luz (claros) y en la sombra (sotobosque) refleja dos tipos de estrategia de defenderse de los herbívoros. Las especies persistentes en el sotobosque crecen despacio y probablemente no pueden permitirse de cambiar las hojas frecuentemente. Por eso, es imprescindible de defender 
las hojas efectivamente contra los herbívoros (Coley 1982). Debido a esta defensa química, la cantidad de epífilas en las hojas de estas especies está reducida significativamente (Coley $e t$ al. 1993). Por el contrario, las especies pioneras dependen de una germinación en claros nuevos. Estas plantas crecen rápidamente y no gastan energía en hojas longevas (Coley 1982).

No existen datos sobre la influencia de la humedad del aire sobre la longevidad de hojas. Se puede suponer, que las hojas en sitios secos son más duras o escleromórficas y por eso son más longevas que hojas en sitios más húmedos.

Además, la longevidad de las hojas tiene importancia para el desarollo de muchos organismos que viven en la superficie foliar. Algunas especies de líquenes de los géneros Porina, Dimerella y Echinoplaca se pueden observar a partir de 2.5 meses (Hartmann 1993). Para frutificar necesitan por lo menos seis meses (E. Freiberg 1994), y la mayoría de las especies necesitan mucho más tiempo para su desarro1lo. En la RBAMB, los musgos aparecen en hojas a partir de cinco meses, y $2-5 \%$ de la superficie de hojas de 7-10 meses de edad es poblada por musgos. Algas azules (cianobacterias) necesitan como mínimo ocho meses para establecerse (E. Freiberg 1994).

Richards (1966) observó que los árboles caducifolios también son abundantes en bosques húmedos con un clima constante, especialmente en el dosel, y que las plantas del estrato arbustivo son casi completamente perennifolios. Los datos de este estudio muestran que aunque el clima en el sotobosque de la RBAMB es relativamente constante, las variaciones mínimas del microclima son suficientes para influir en el crecimiento de las plantas en el estrato arbustivo.

\section{AGRADECIMIENTOS}

Agradezco a Rodolfo Ortiz Vargas, director de la Reserva Biológica Alberto Ml. Brenes, a Victor Mora y la Universidad de Costa Rica y el Herbario Nacional de Costa Rica por su apoyo. Además, doy las gracias a Alma
Koch y Klaus Mehltreter por sus comentarios sobre el texto y por su ayuda en corregir el manuscrito. Esta investigación recibió apoyo a través de fondos del DAAD (Servicio Alemán de Intercambio Académico) y de la Studienstiftung des Deutschen Volkes.

\section{RESUMEN}

Durante un año y medio se investigó el ritmo de crecimiento y la longevidad de follaje de 22 especies abundantes del estrato arbustivo en el bosque tropical premontano de la Reserva Biológica Alberto Ml. Brenes, Provincia Alajuela, San Ramón, Costa Rica. Se distinguieron y describieron tres tipos de brotación de las hojas. Una gran cantidad de las especies, especialmente las monocotiledóneas, brotan hojas nuevas en intervalos determinados contínuamente durante todo el año, en efecto parte de las plantas muestran una producción de hojas más lenta en la estación seca. Esta frecuencia de brotación, es decir el intervalo entre el desarrollo de dos hojas siguientes, varía entre las especies investigadas entre 17-22 días y más de medio año. La longevidad de follaje promedio de las plantas investigadas varía entre medio y cuatro años. Se estimó que la edad máxima de hojas de las palmas Cryosophila warscewiczii, Iriartea deltoidea y Calyptrogyne trichostachys es más de cinco años. Generalmente, en sitios más secos y más oscuros, la frecuencia de brotación fue más larga y la longevidad de follaje más alta. Esta relación fue significante en algunos casos, pero muestra que aunque las diferencias microclimáticas son muy pequeñas, el crecimiento varía entre diferentes sitios en el bosque. Además, los datos muestran que aunque el clima en el sotobosque premontano muy húmedo es relativamente constante, algunas especies muestran una estacionalidad del ritmo de crecimiento.

\section{REFERENCIAS}

Ackerly, D.D. \& F.A. Bazzaz. 1995. Leaf dynamics, selfshading and carbon gain in seedlings of a tropical pioneer tree. Oecologia 101: 289-298.

Addicott, F.T. \& J.L. Lyon. 1973. Physiological ecology of abscission. In T.T. Kozlowski (ed.). The Shedding of Plant Parts. Academic, Londres. 560 p.

Bannister, P. 1986. Water relation and stress, p. 73-82. In P.D. Moore \& S.B. Chapman (eds.). Methods in Plant Ecology. Blackwell Scientific, Oxford.

Bentley, B.L. 1979. Longevity of individual leaves in a tropical rainforest understory. Ann. Bot. 43: 119121. 
Borchert, R. 1983. Phenology and control of flowering in tropical trees. Biotropica 15: 81-89.

Chabot, B.F. \& D.J. Hicks. 1982. The ecology of leaf life spans. Annu. Rev. Ecol. Syst. 13: 229-259.

Clark, D.B., Clark, D.A. \& Grayum, M.H. (1992): Leaf demography of a neotropical rain forest cycad, $\mathrm{Za}$ mia skinneri (Zamiaceae). Am. J. Bot. 78: 28-33.

Coley, P.D. 1982. Rates of herbivory on different tropical trees, p. 123-132. In E.G.Jr. Leigh, A.S. Rand \& D.M. Windsor (eds.). The Ecology of a Tropical Forest. Seasonal Rhythms and Long-term Changes. Smithsonian Institution, Washington, D.C.

Coley, P.D. 1988. Effects of plant growth rate and leaf lifetime on the amount and type of anti-herbivore defense. Oecologia 74: 531-536.

Coley, P.D., T.A. Kursar \& J.-L. Machado. 1993. Colonization of tropical rain forest leaves by epiphylls: effects of site and host plant leaf lifetime. Ecology 74: 619-623.

Dombeck, T. 1997. Distribución altitudinal de los helechos arborescentes en la Reserva Biológica Alberto Brenes, Cordillera de Tilarán, p. 37-45. In I. Wattenberg \& S.-W. Breckle (eds). Contributions to Tropical Ecology Research in Costa Rica. Bielfelder Ökologische Beiträge 11.

Frankie, G.W., H.G. Baker \& P.A. Opler. 1974. Comparative phenological studies of trees in tropical wet and dry forests in the lowlands of Costa Rica. J. Ecol. 62 881-913.

Freiberg, E. 1994. Stickstoffixierung in der Phyllosphäre tropischer Regenwaldpflanzen in Costa Rica. Tesis de doctorado, Universidad de Ulm, Alemania. 121 p.

Freiberg, E. 1998. Microclimatical parameters influencing nitrogen fixation in the phyllosphere in a Costa $\mathrm{Ri}$ can premontane rain forest. Oecologia 117: 9-18.

Freiberg, M. 1994. Phänomorphologie epiphytischer Gesneriaceen in Costa Rica unter besonderer Berücksichtigung des Mikroklimas. Tesis de doctorado, Universidad de Ulm, Alemania. 147 p.

Freiberg, M. 1996. Phenotype expression of epipyhtic Gesneriaceae under different microclimatic conditions in Costa Rica. Ecotropica 2: 49-57.

Freiberg, M. 1997. Spatial and temporal pattern of temperature and humidity of a tropical premontane rain forest tree in Costa Rica. Selbyana 18: 77-84.
Hartmann, C. 1993. Ökologische Untersuchungen an foliikolen Flechten in Costa Rica, Zentralamerika. Tésis de diploma, Universidad de Ulm, Alemania. $86 \mathrm{p}$.

Härtshorn, G.S. 1983. Costa Rica Perfil Ambiental. Centro Científico Tropical, San José, Costa Rica. 151 p.

Holdridge, L.R. 1967. Life Zone Ecology. Tropical Science Center, San José, Costa Rica. 206 p.

Janzen, D.H. 1975. Ecology of Plants in the Tropics. Butler \& Tanner, Frome, Londres. 66 p.

Leigh, E.G.Jr. \& D.M. Winsdor. 1982. Forest production and regulation of primary consumers on Barro Colorado Island, p. 111-122. In E.G.Jr. Leigh, A.S. Rand \& D.M. Windsor (eds.). The Ecology of a Tropical Forest. Seasonal Rhythms and Long-term Changes. Smithsonian Institution, Washington, D.C.

Lücking, A. 1995. Diversität und Mikrohabitatpräferenzen epiphyller Moose in einem tropischen Regenwald in Costa Rica unter besonderer Berücksichtigung der Familie Lejeuneaceae. Tesis de doctorado, Universidad de Ulm, Alemania. $211 \mathrm{p}$

Lücking, R. 1994. Foliikole Flechten und ihre Mikrohabitatpräferenzen in einem tropischen Regenwald in Costa Rica. Tesis de doctorado, Universidad de Ulm, Alemania. 203 p.

Mabberly, D.J. 1983. Tropical Rain Forest Ecology. Blackie, Bishopbriggs, Glasgow. 156 p.

Medway. L. (1972): Phenology of a tropical rain forest in Malaya. Biol. J. Linnean Soc., London 4: 117-146.

Ortiz Vargas, R. (ed.). 1991. Memoria de Investigación Reserva Forestal de San Ramón. Universidad de Costa Rica (Serie Catédra Universitaria), San José, Costa Rica. 110 p.

Reich, P.B. \& R. Borchert. 1984. Water stress and tree phenology in a tropical dry forest in the lowlands of Costa Rica. J. Ecol. 72: 61-74.

Richards, P.W. 1966. The tropical rain forest. An ecological study. Cambridge University, Cambridge. $450 \mathrm{p}$.

Vargas, G. 1991. Algunas consideraciones geográficas, geológicas y ecológicas de la cienca del Río San Lorenzo, San Ramón, Alajuela, Costa Rica, p. 17-22. In Ortiz Vargas. R. (ed.). Memoria de Investigación. Reserva Forestal San Ramón. Universidad de Costa Rica (Serie Catédra Universitaria), San José, Costa Rica. 
Walter, H. (1973): Die Vegetation der Erde. Band I. Die tropischen und subtropischen Zonen. Gustav Fischer, Stuttgart. 743 p.

Wattenberg, I. \& S.-W. Breckle. 1995. Tree species diversity of a premontane rain forest in the Cordillera de Tilarán, Costa Rica. Ecotropica 1: 21-30.
Wattenberg, I. \& S.-W. Breckle (eds.). 1997. Contributions to Tropical Ecology Research in Costa Rica. Bielfelder Ökologische Beiträge, vol. 11, Bielefeld, $99 \mathrm{p}$.

Whitmore, T.C. 1993. Tropische Regenwälder. Spektrum Akademischer, Heidelberg. 275 p. 